\title{
Global branches of positive weak solutions of semilinear elliptic problems over nonsmooth domains
}

\author{
Timothy J. Healey \\ Department of Theoretical \& Applied Mechanics and Center for Applied \\ Mathematics, Cornell University, Ithaca, NY 14853, U.S.A. \\ Hansjörg Kielhöfer \\ Institut für Mathematik, Universität Augsburg, Universitätsstraße 8, \\ D-8900 Augsburg, Germany
}

\author{
Charles A. Stuart \\ Départment de Mathématiques, Ecole Polytechnique Fédérale de \\ Lausanne, CH-1015 Lausanne, Switzerland
}

(MS received 5 February 1992. Revised MS received 10 June 1993)

\begin{abstract}
We consider the nonlinear eigenvalue problem posed by a parameter-dependent semilinear second-order elliptic equation on a bounded domain with the Dirichlet boundary condition. The coefficients of the elliptic operator are bounded measurable functions and the boundary of the domain is only required to be regular in the sense of Wiener. The main results establish the existence of an unbounded branch of positive weak solutions.
\end{abstract}

\section{Introduction}

Let $\Omega$ be a bounded domain (nonempty open connected subset) in $\mathbb{R}^{n}$. We consider weak (or generalised) solutions of the semilinear boundary-value problem

$$
\begin{aligned}
L u(x)+f(x, u(x), \lambda)=0 & & \text { for } x \in \Omega, \\
u(x)=0 & & \text { for } x \in \partial \Omega,
\end{aligned}
$$

where $L$ is a second-order elliptic operator with bounded measurable coefficients and, for each $\lambda \in \mathbb{R}, f$ is a function of Carathéodory type. Concerning the boundary $\partial \Omega$, we assume just enough regularity to ensure that weak solutions of (1.1) belong to $W_{0}^{1,2}(\Omega) \cap C(\bar{\Omega})$ and fulfill the Dirichlet boundary condition pointwise. (For example, the requirement that every point of $\partial \Omega$ satisfies an exterior cone condition is sufficient.) In this setting we discuss the global behaviour of the principal component $C$ of weak solution pairs $(\lambda, u)$ of $(1.1)$.

We treat two common situations:

(i) (Bifurcation) We assume that $f(x, 0, \lambda) \equiv 0$ for all $\lambda \in \mathbb{R}$, in which case $C$ denotes the branch of solutions bifurcating from the line of trivial solutions, $\mathbb{R} \times\{0\}$, at a principal eigenvalue of the linearisation of (1.1). 
(ii) (Continuation) We take $f(x, 0,0)=0$ and then $C$ is the solution branch containing $(0,0) \in \mathbb{R} \times C(\bar{\Omega})$.

In both cases our main result is that for non-trivial elements $(\lambda, u) \in C$ the solution $u$ must be of one sign on $\Omega$.

In the pioneering work of Rabinowitz $[9,10]$, a similar result was obtained for regular problems (1.1) on regular domains via the Hopf maximum principle. Specifically, for nontrivial elements of $C$, it is shown that $u \in K$ (or $-u \in K$ ), where $K$ is the open cone

$$
K=\left\{u \in C^{1}(\bar{\Omega}): u>0 \text { in } \Omega, \frac{\partial u}{\partial n}<0 \text { and } u=0 \text { on } \partial \Omega\right\} .
$$

(Here $\partial u / \partial n$ denotes the outer normal derivative of $u$.) Clearly, in our situation, solutions of (1.1) need not have $C^{1}(\bar{\Omega})$ regularity, and the cone of positive functions in $W_{0}^{1,2}(\Omega) \cap C(\bar{\Omega})$ is not open. Consequently, the arguments employed in $[9,10]$ are not readily generalised to the problem at hand. We overcome this difficulty via another refinement of the maximum principle that was first introduced in the context of regular versions of (1.1) on polygonal domains with corners in the presence of symmetries [6].

The plan of the paper is as follows. In Section 2 we state our basic assumptions on $L$ and $f$ in (1.1), and then recall a few well-known consequences. We then establish a refinement of the maximum principle in Section 3. In Section 4 we reformulate our generalised problem as an operator equation in the form of a compact perturbation of the identity; this is crucial to the global bifurcation and global continuation analyses presented in Sections 5 and 6, respectively. In Section 7 we indicate the applicability of the results from Section 6 to the anti-plane-shear problem of nonlinear elasticity.

The main results of the paper are presented in Sections 5 and 6: we establish the positivity (negativity) of generalised solutions along global solution branches. A novel feature of our treatment of the bifurcation case in Section 5, is that our results hold with a weak odd-crossing hypothesis that ensures the existence of global continua of nontrivial solutions. Moreover, in the continuation case, presented in Section 6, we separate the zero-order perturbation into the sum of a nonlinear part and an inhomogeneous "forcing" term. In this way, we are able to place separate hypotheses upon each term. In particular, our results hold for each of two distinct hypotheses concerning the (local) sign of the nonlinear term (cf. (6.6) and (6.7)).

Notation 1.1. For $1 \leqq p \leqq \infty$ and $\Omega$ a bounded open subset of $\mathbb{R}^{n}$, the usual norm on the space $L^{p}(\Omega)$ will be denoted by $\|\cdot\|_{p}$ or \|\|$_{L^{p}(\Omega)}$, as the context requires. Similarly, the usual norm on $C(\bar{\Omega})$ will be expressed as $\|\cdot\|_{\infty}$.

\section{Preliminaries}

In this section we define the linear operator $L$ and the nonlinearity $f$. Let $\Omega$ be a bounded domain in $\mathbb{R}^{n}$. Using the summation convention, we define a differential operator $L$ on $\Omega$ by

$$
L u(x)=D_{i}\left\{a^{i j}(x) D_{j} u(x)+b^{i}(x) u(x)\right\}+c^{i}(x) D_{i} u(x)+d(x) u(x),
$$


where the coefficients satisfy the following conditions:

$$
\left.\begin{array}{l}
\text { (i) } a^{i j}=a^{j i}, b^{i}, c^{i}, d \in L^{\infty}(\Omega) \text { for } i, j \in\{1, \ldots, n\} \text {; } \\
\text { (ii) there is an } \alpha>0 \text { such that } \\
a^{i j}(x) \xi_{i} \xi_{j} \geqq \alpha|\xi|^{2} \text { for almost all } x \in \Omega, \xi \in \mathbb{R}^{n} \text {; } \\
\text { (iii) } \int_{\Omega}\left(d v-b^{i} D_{i} v\right) d x \leqq 0 \text { for all non-negative } v \in W^{1,1}(\Omega) \text {. }
\end{array}\right\}
$$

REMARK 2.1. By (2.2(ii)), $L$ is uniformly elliptic on $\Omega$. We can extend $L$ to an operator $\hat{L}$ having the same properties on all $\mathbb{R}^{n}$ by setting $a^{i j}(x)=\alpha \delta_{i j}, b^{i}(x)=c^{i}(x)=d(x)=$ 0 for $x \in \mathbb{R}^{n} \backslash \Omega, 1 \leqq i, j \leqq n$. Then inequality (2.2(iii)) holds for all non-negative $v \in W^{\mathbf{1}, 1}\left(\mathbb{R}^{n}\right)$. It is for this reason that we have replaced the usual condition $([\mathbf{5}$, (8.8)] by the stronger form (2.2(iii)).

For the nonlinearity we consider a function

$$
f: \Omega \times \mathbb{R}^{2} \rightarrow \mathbb{R} \text { of the form } f(x, s, \lambda)=g(x, \lambda)+h(x, s, \lambda) s \text {, where }
$$

(i) $f(\cdot, s, \lambda): \Omega \rightarrow \mathbb{R}$ is measurable for all $(s, \lambda) \in \mathbb{R}^{2}$;

(ii) for almost all $x \in \Omega, g(x, \cdot): \mathbb{R} \rightarrow \mathbb{R}$ and $h(x, \cdot, \cdot): \mathbb{R}^{2} \rightarrow \mathbb{R}$ are continuous;

(iii) there is a $\delta>0$ such that for almost all $x \in \Omega$ the partial derivative of $f(x, \cdot, \cdot)$ with respect to $s$ exists and is continuous on $V$ where $V=(-\delta, \delta) \times \mathbb{R}$

(iv) the functions $g, h, f_{s}$ are bounded on bounded subsets of their domains of definition, respectively.

For $(\lambda, u) \in \mathbb{R} \times C(\bar{\Omega})$, we introduce

$$
\begin{aligned}
F(\lambda, u)(x) & \equiv f(x, u(x), \lambda), \\
A(\lambda) u(x) & \equiv h(x, 0, \lambda) u(x) .
\end{aligned}
$$

By standard arguments we then obtain the following results:

Proposition 2.2. Let $\Omega$ be a bounded domain in $\mathbb{R}^{n}$ and let $p \in[1, \infty)$. Suppose that $f$ satisfies all of (2.3).Then the mapping $F$ as defined in (2.4) has the following properties:

(i) $F: \mathbb{R} \times C(\bar{\Omega}) \rightarrow L^{p}(\Omega)$ is bounded and continuous;

(ii) $A: \mathbb{R} \rightarrow \mathscr{L}\left(C(\bar{\Omega}), L^{p}(\Omega)\right)$ is continuous, where $\mathscr{L}$ denotes the space of linear and bounded operators;

(iii) $F: \mathbb{R} \times C(\bar{\Omega}) \rightarrow L^{p}(\Omega)$ is continuously Fréchet differentiable with respect to $u$ at all $(\lambda, u) \in \mathbb{R} \times C(\bar{\Omega})$ such that $\|u\|_{\infty}<\delta$, and $D_{u} F(\lambda, 0)=A(\lambda)$.

By imposing further restrictions on $f$ we can also ensure that $F$ is twice continuously differentiable with respect to $\lambda$ and $u$ near $(\lambda, 0)$.

Proposition 2.3. Let $\Omega$ be a bounded domain in $\mathbb{R}^{n}$ and let $p \in[1, \infty)$. In addition to the hypotheses (2.3), suppose that $f$ satisfies:

there is a $\delta>0$ such that $f(x, \cdot, \cdot) \in C^{2}(V)$ for almost all $x \in \Omega$, where $V=(-\delta, \delta) \times \mathbb{R}$, and $D_{m} f$ is bounded on bounded subsets of $\Omega \times V$, where $D_{m} f$ denotes any partial derivative of order $\leqq 2$ with respect to 
$\lambda$ and $s$.

Then $F: \mathbb{R} \times C(\bar{\Omega}) \rightarrow L^{p}(\Omega)$ is twice continuously Fréchet differentiable on $\left\{(\lambda, u) \in \mathbb{R} \times C(\bar{\Omega}):\|u\|_{\infty}<\delta\right\}$. In particular,

$$
D_{\lambda} D_{u} F(\lambda, 0) v(x)=\frac{\partial h}{\partial \lambda}(x, 0, \lambda) v(x)
$$

for $(\lambda, v) \in \mathbb{R} \times C(\bar{\Omega})$ and $x \in \Omega$.

\section{A maximum principle}

In this section we formulate a version of the maximum principle for $L$. In connection with components of solutions of (1.1), it was first introduced in [6] to deal with regular problems on polygonal domains in the presence of the symmetry of a lattice in $\mathbb{R}^{n}$. We first need the following well-known result (cf. [3]. Theorem IX.17] or [6, Appendix]):

Lemma 3.1. Let $\Omega$ be a bounded open subset of $\mathbb{R}^{n}$. Suppose that $u \in W^{1.2}(\Omega) \cap C(\bar{\Omega})$ and that $u(x)=0$ for all $x \in \partial \Omega$. Then $u \in W_{0}^{1,2}(\Omega)$.

Proof. This is the implication (i) $\Rightarrow$ (ii) of [3, Theorem IX.17]. We note that the proof of this assertion does not require any restriction on the boundary of $\Omega$.

THEOREM 3.2. Let $\Omega$ be a bounded domain in $\mathbb{R}^{n}$ and let $L$ satisfy the conditions (2.2). Suppose that $r \in L^{\infty}(\Omega)$ and that $v \in W_{0}^{1.2}(\Omega) \cap C(\bar{\Omega}) \backslash\{0\}$ satisfies

$$
L v+r v \leqq 0 \text { in } \Omega
$$

in the generalised sense (cf. [5, equation (8.2)]. Let $s^{+}=\max \{s, 0\}$ for $s \in \mathbb{R}$. Then there exists a number $\gamma>0$, depending only upon $\max _{i}\left\|b^{i}-c^{i}\right\|_{\infty},\left\|(d+r)^{+}\right\|_{\infty}, \alpha$, and $n$, such that

$$
\text { meas }\{x \in \Omega: v(x)<0\}<\gamma
$$

implies $v(x)>0$ for all $x \in \Omega$.

Proof. Let $v^{-}=\min \{v, 0\}$. Then by $\left[5\right.$, Lemma 7.6] $v^{-} \in W^{1,2}(\Omega)$. Setting $\mathscr{D}=$ $\{x \in \Omega, v(x)<0\}$, we suppose that $\mathscr{D} \neq \varnothing$. Then $v^{-} \in W_{0}^{1,2}(\mathscr{D}) \cap C(\mathscr{D})$ since $v^{-}=0$ on $\partial \mathscr{D}$ (see Lemma 3.1). By Poincaré's inequality (cf. [5, equation (7.44)]).

$$
\left\|v^{-}\right\|_{L^{2}(\mathscr{O})} \leqq\left(\frac{|\mathscr{D}|}{\omega_{n}}\right)^{1 / n}\left\|\nabla v^{-}\right\|_{L^{2}(\mathscr{D})} \quad\left(\omega_{n}=\text { volume of unit ball }\right) .
$$

Since $v^{-}=0$ and $\nabla v^{-}=0$ almost everywhere on $\Omega \backslash \mathscr{D}$, this yields

$$
\left\|v^{-}\right\|_{L^{2}(\Omega)} \leqq\left(\frac{|\mathscr{D}|}{\omega_{n}}\right)^{1 / n}\left\|\nabla v^{-}\right\|_{L^{2}(\Omega)}
$$

Since $v^{-} \in W_{0}^{1,2}(\Omega)$ and $v^{-} \leqq 0$ in $\Omega$, it follows from the hypothesis $L v+r v \leqq 0$ in $\Omega$ (in the generalised sense), that

$$
\int_{\Omega}\left\{a^{i j} D_{j} v+b^{i} v\right\} D_{i} v^{-}-\left(c^{i} D_{i} v+(d+r) v\right) v^{-} d x \leqq 0 .
$$


Consequently,

$$
\begin{aligned}
& \int_{\Omega} a^{i j} D_{j} v^{-} D_{i} v^{-} d x \leqq \int_{\Omega}\left(c^{i}-b^{i}\right) v^{-} D_{i} v^{-}+(d+r)\left(v^{-}\right)^{2} d x \\
& \quad \leqq\left\|b^{i}-c^{i}\right\|_{L^{\infty}(\Omega)}\left(\frac{\varepsilon}{2}\left\|D_{i} v^{-}\right\|_{L^{2}(\Omega)}^{2}+\frac{1}{2 \varepsilon}\left\|v^{-}\right\|_{L^{2}(\Omega)}^{2}\right)+\left\|(d+r)^{+}\right\|_{L^{\infty}(\Omega)}\left\|v^{-}\right\|_{L^{2}(\Omega)}^{2} \\
& \quad \leqq \frac{M \varepsilon}{2}\left\|\nabla v^{-}\right\|_{L^{2}(\Omega)}^{2}+\left(\frac{M n}{2 \varepsilon}+\left\|(d+r)^{+}\right\|_{L^{\infty}(\Omega)}\right)\left\|v^{-}\right\|_{L^{2}(\Omega)}^{2}
\end{aligned}
$$

where $M=\max _{i}\left\|b^{i}-c^{i}\right\|_{L^{\infty}(\Omega)}$.

Using uniform ellipticity (2.2(ii)), we then obtain

$$
\alpha\left\|\nabla v^{-}\right\|_{L^{2}(\Omega)}^{2} \leqq \frac{M \varepsilon}{2}\left\|\nabla v^{-}\right\|_{L^{2}(\Omega)}^{2}+\left(\frac{M n}{2 \varepsilon}+\left\|(d+r)^{+}\right\|_{L^{\infty}(\Omega)}\right)\left\|v^{-}\right\|_{L^{2}(\Omega)}^{2},
$$

and choosing $\varepsilon=\alpha / M$ this yields

$$
\frac{\alpha}{2}\left\|\nabla v^{-}\right\|_{L^{2}(\Omega)}^{2} \leqq\left(\frac{M^{2} n}{2 \alpha}+\left\|(d+r)^{+}\right\|_{L^{\infty}(\Omega)}\right)\left\|v^{-}\right\|_{L^{2}(\Omega)}^{2}
$$

Recalling (3.2), we have

$$
\left(\frac{\omega_{n}}{|\mathscr{D}|}\right)^{2 / n} \frac{\alpha}{2}\left\|v^{-}\right\|_{L^{2}(\Omega)}^{2} \leqq\left(\frac{M^{2} n}{2 \alpha}+\left\|(d+r)^{+}\right\|_{L^{\infty}(\Omega)}\right)\left\|v^{-}\right\|_{L^{2}(\Omega)}^{2} .
$$

Hence, since $\mathscr{D} \neq \varnothing$ implies $\left\|v^{-}\right\|_{L^{2}(\Omega)} \neq 0$, it follows that

$$
|\mathscr{D}| \geqq \omega_{n}\left(\frac{M^{2} n}{\alpha^{2}}+\frac{-2}{\alpha}\left\|(d+r)^{+}\right\|_{L^{\infty}(\Omega)}\right)^{-n / 2} \equiv \gamma
$$

With that definition of $\gamma$, it follows that either $|\mathscr{D}| \geqq \gamma$ or $\mathscr{D}=\varnothing$. Hence, if $|\mathscr{D}|<\gamma$, then $v \geqq 0$ on $\Omega$. But then

$$
0 \geqq L v+r v=L v+r^{-} v+r^{+} v \geqq L v+r^{-} v \text { on } \Omega
$$

in the generalised sense. Since $L+r^{-}$satisfies all three conditions in (2.2), it follows from [5, Theorem 8.19] and the connectedness of $\Omega$ that either $v \equiv 0$ on $\Omega$ or $v(x)>0$ for all $x \in \Omega$.

REMARK 3.3. We emphasise that Theorem 3.2 holds without imposing any sign condition on the function $r$. This follows immediately from inequality (3.9), which was first observed by Serrin [12].

\section{Problem formulation}

Let $L$ be an elliptic operator satisfying conditions (2.2) and let $f$ be a function having the properties (2.3).

Definition 4.1. By a generalised (or weak) solution of the nonlinear problem

$$
\begin{aligned}
L u(x)+f(x, u(x), \lambda)=0 & & \text { for } x \in \Omega, \\
u(x)=0 & & \text { for } x \in \partial \Omega,
\end{aligned}
$$


we mean a pair $(\lambda, u) \in \mathbb{R} \times\left(W_{0}^{1,2}(\Omega) \cap C(\bar{\Omega})\right)$ such that

$$
\int_{\Omega}\left\{a^{i j} D_{j} u+b^{i} u\right\} D_{i} v-\left(c^{i} D_{i} u+d u+f(\cdot, u, \lambda)\right) v d x=0 \text { for all } v \in C_{0}^{\infty}(\Omega)
$$

This definition is apparently not adequate for a global bifurcation continuation analysis. To this end, we now pursue a more convenient formulation of our problem. As a first step we consider the following linear problem:

Definition 4.2. For $g \in L^{2}(\Omega)$, by a weak or generalised solution of the linear Dirichlet problem

$$
\begin{aligned}
L u(x)+g(x)=0 & \text { for } x \in \Omega, \\
u(x)=0 & \text { for } x \in \partial \Omega,
\end{aligned}
$$

we mean a function $u \in W_{0}^{1,2}(\Omega)$ such that

$$
\int_{\Omega}\left\{a^{i j} D_{j} u+b^{i} u\right\} D_{i} v-\left(c^{i} D_{i} u+d u+g\right) v d x=0 \text { for all } v \in C_{0}^{\infty}(\Omega) .
$$

Proposition 4.3. Let $g \in L^{2}(\Omega)$. Then there exists a unique generalised solution $u$ of the Dirichlet problem (4.3). (See [5, Theorem 8.3])

Definition 4.4. For $g \in L^{2}(\Omega)$, we denote the unique solution $u$ of (4.4) by $T g$. Hence $T$ is a linear operator from $L^{2}(\Omega)$ into $W_{0}^{1,2}(\Omega)$.

The crucial step in our reformulation of the nonlinear problem is based on the following properties of $T$. To obtain them, we impose a well-known restriction on the boundary of $\Omega$.

$\Omega$ is a bounded domain in $\mathbb{R}^{n}$ such that every point $x \in \partial \Omega$ is regular in the sense of Wiener for Laplace's equation.

(For a definition, see [5, Chapter 2.8]). A necessary and sufficient condition for (4.5) is the so-called Wiener criterion which states that the series (2.37) of [5] diverges. A sufficient condition for (4.5) is that

$\Omega$ satisfies an exterior cone condition

(see [5, Problem 2.12]). In two dimensions $(n=2)$, the conditions on $\partial \Omega$ can be considerably generalised (see [5, Chapter 2.8]). The subsequent discussion is throughout based on assumption (4.5).

THEOREM 4.5. Let $p \in[2, \infty) \cap(n / 2, \infty)$.

(i) For $g \in L^{p}(\Omega), \operatorname{Tg} \in C(\bar{\Omega})$ with $\operatorname{Tg}(x)=0$ for all $x \in \partial \Omega$.

(ii) The linear operator $T: L^{p}(\Omega) \rightarrow C(\bar{\Omega})$ is compact.

Proof. Let $L_{1}$ be the operator defined by $L_{1} u=L u-\xi u$, where $\xi=\left\|d^{+}\right\|_{L^{\infty}(\Omega)}+1$. Clearly $L_{1}$ satisfies $(2.2)$ and $d_{1}(x) \equiv d(x)-\xi \leqq-1$ on $\Omega$. For $g \in L^{2}(\Omega)$, let $T_{1} g \in W_{0}^{1,2}(\Omega)$ denote the unique generalised solution of that Dirichlet problem $L_{1} u(x)+g(x)=0$ in $\Omega, u(x)=0$ on $\partial \Omega$, that is given by Proposition 4.3. Suppose now that $g \in L^{p}(\Omega)$ and let us consider $T g$ and $T_{1} g$. Clearly $g+\xi T g \in L^{2}(\Omega)$, and a simple calculation shows that $T g=T_{1}(g+\xi T g)$. 
Furthermore, by [5, Theorem 8.16], $\operatorname{Tg} \in L^{\infty}(\Omega)$ and

$$
T: L^{p}(\Omega) \rightarrow L^{\infty}(\Omega) \text { is a bounded linear operator. }
$$

Now let $B$ be an open ball in $\mathbb{R}^{n}$ such that $\bar{\Omega} \subset B$. We define a bounded linear operator

$$
E: L^{p}(\Omega) \rightarrow L^{p}(B) \quad \text { by } \quad E g(x)=g(x) \quad \text { for } x \in \Omega, \quad E g(x)=0 \quad \text { for } x \in B \backslash \Omega .
$$

Let $\hat{L}_{1}$ be defined by $\hat{L}_{1} u=\hat{L} u-\xi u$, where $\hat{L}$ is the extension of $L$ to $B$ discussed in Remark 2.1. For $h \in L^{p}(B)$, let $\hat{T}_{1} h \in W_{0}^{1,2}(B)$ be the unique generalised solution of the Dirichlet problem

$$
\begin{aligned}
\hat{L}_{1} u(x)+h(x)=0 & \text { for } x \in B, \\
u(x)=0 & \text { for } x \in \partial B .
\end{aligned}
$$

Then, by [5, Theorem 8.22], $\hat{T}_{1} h \in C(B)$ and by [5, Theorem 8.24], $\hat{T}_{1} h$ is in some Hölder space $C^{\mu}(\bar{\Omega})$ for $\mu \in(0,1)$ and there is some constant $C_{1}>0$ such that

$$
\left\|\hat{T}_{1} h\right\|_{C^{\mu}(\bar{\Omega})} \leqq C_{1}\left(\left\|\hat{T}_{1} h\right\|_{L^{2}(B)}+\|h\|_{L^{p}(B)}\right) .
$$

By $[5$, Corollary 8.7$]$, there is some $C_{2}>0$ such that

$$
\left\|\hat{T}_{1} h\right\|_{L^{2}(B)} \leqq C_{2}\|h\|_{L^{2}(B)},
$$

and hence there exists $C_{3}>0$ such that

$$
\left\|\hat{T}_{1} h\right\|_{C^{\mu}(\hat{\mathbf{\Omega}})} \leqq C_{3}\|h\|_{L^{p}(\boldsymbol{B})} .
$$

Next we consider the Dirichlet problem

$$
\begin{array}{ll}
L_{1} w(x)=0 & \text { for } x \in \Omega, \\
w(x)=\varphi(x) & \text { for } x \in \partial \Omega,
\end{array}
$$

where $\varphi \in W_{0}^{1,2}(B) \cap C(B)$. Using [5, Theorem 8.3], there is a unique generalised solution $w \in W^{1,2}(\Omega)$ of (4.13) satisfying the boundary condition in the sense that $w-\varphi \in W_{0}^{1,2}(\Omega)$. We denote this solution by $S \varphi$. By the assumed regularity of the boundary data $\varphi$, the uniqueness implies that this solution $w$ coincides with a (generalised) solution $w=B \varphi \in H^{1}(\Omega)$ of (4.13) in the sense of [13, Chapter 10$]$. (Observe that the hypotheses $(1.2),(9.1),(9.2)$ of $[13]$ are satisfied by $L_{1}$.) By [13, Theorem 7.1] (or [5, Theorem 8.22]) $w=S \varphi \in C(\Omega)$ and, by $[13,(10.1)]$ (or by [5, Theorem 8.1$]$ ), we also have that

$$
\sup _{x \in \mathbf{\Omega}}|S \varphi(x)| \leqq \max _{x \in \partial \boldsymbol{\Omega}}|\varphi(x)|
$$

Finally, since by (4.5) every point $x_{0} \in \partial \Omega$ is regular (in the sense of Wiener) for Laplace's equation on $\Omega,[13$, Theorem 10.2] yields

$$
\lim _{x \rightarrow x_{0}} S \varphi(x)=\varphi\left(x_{0}\right) \quad \text { for all } x_{0} \in \partial \Omega
$$

In other words: $S \varphi \in C(\bar{\Omega})$ and $(S \varphi-\varphi)(x)=0$ for all $x \in \partial \Omega$. Now for $g \in L^{p}(\Omega)$ we set

$$
w=R g-S R g \quad \text { where } R g=\hat{T}_{1} E(g+\xi T g)
$$


From the preceding observations, $R g \in W^{1,2}(\Omega) \cap C^{\mu}(\bar{\Omega})$ and $S R g \in W^{1,2}(\Omega) \cap C(\bar{\Omega})$. Hence, by (4.15), Lemma 3.1 and (4.7), $w \in W_{0}^{1,2}(\Omega) \cap \mathrm{C}(\bar{\Omega}), w(x)=0 \quad$ for all $x \in \partial \Omega, \quad$ and $\quad w=T_{1}(g+\xi T g)=T g$.

This proves the first part of Theorem 4.5.

To prove the compactness of $T$, consider a sequence $\left(g_{n}\right)$ that is bounded in $L^{p}(\Omega)$. By (4.7) $\left(g_{n}+\xi T g_{n}\right)$ is a bounded sequence in $L^{p}(\Omega)$ as well. By (4.12) $\left(R g_{n}\right)$ is a bounded sequence in $C^{\mu}(\bar{\Omega})$ and so, by the Theorem of Ascoli and Arzela, there is a subsequence such that $\left(R g_{n_{k}}\right)$ also converges in $C(\bar{\Omega})$. Then (4.14) implies that $\left(S R g_{n_{k}}\right)$ converges in $C(\bar{\Omega})$. Since $T g_{n_{k}}=R g_{n_{k}}-S R g_{n_{k}}$, we have established the compactness of $T: L^{p}(\Omega) \rightarrow C(\bar{\Omega})$.

We can now restate the nonlinear problem (4.1) in a form amenable to a global analysis (for the operator $F$ see Proposition 2.2).

Proposition 4.6. A pair $(\lambda, u)$ is a generalised solution of the nonlinear problem (4.1) if and only if $(\lambda, u) \in \mathbb{R} \times C(\bar{\Omega})$ solves $u=T F(\lambda, u)$. This, in turn, is equivalent to the equation

$$
G(\lambda, u)=0,
$$

where $G: \mathbb{R} \times C(\bar{\Omega}) \rightarrow C(\bar{\Omega})$ is defined by $G(\lambda, u)=u-K(\lambda, u)$ with $K(\lambda, u)=T F(\lambda, u)$. Moreover, $K: \mathbb{R} \times C(\bar{\Omega}) \rightarrow C(\bar{\Omega})$ is continuous, bounded and compact. Finally, any fixed point $u=T F(\lambda, u)$ in $C(\bar{\Omega})$ satisfies $u(x)=0$ for all $x \in \partial \Omega$.

\section{Bifurcation}

In this section we study problem (4.18) under the assumptions of Sections 2 and 4, and, in addition, we suppose that

$$
g(x, \lambda)=f(x, 0, \lambda) \equiv 0 \text { for all } x \in \Omega, \lambda \in \mathbb{R} .
$$

This implies $G(\lambda, 0)=0$ for all $\lambda \in \mathbb{R}$, where $\{(\lambda, 0), \lambda \in \mathbb{R}\}$ is commonly called the line of trivial solutions. By Proposition 2.2, the map $G$ is continuously Fréchet differentiable with respect to $u$ near the trivial solution line and $D_{u} G(\lambda, 0)=I-T A(\lambda)$, which is a compact perturbation of the identity. A necessary condition for bifurcation from $\{(\lambda, 0)\}$ at some $\lambda_{0}$ is that $D_{u} G\left(\lambda_{0}, 0\right)$ has a nontrivial kernel: $D_{u} G\left(\lambda_{0}, 0\right) v=0$ for some $v \in C(\bar{\Omega}) \backslash\{0\}$. In other words, $D_{u} G\left(\lambda_{0}, 0\right)$ has eigenvalue zero, which is isolated, by virtue of Riesz-Schauder theory. The relationship of this problem to the linearised differential equation is given below.

Lemma 5.1. The following statements are equivalent:

(a) $(\lambda, v) \in \mathbb{R} \times C(\bar{\Omega})$ satisfies $D_{u} G(\lambda, 0) v=0$.

(b) $(\lambda, v) \in \mathbb{R} \times W_{0}^{1,2}(\Omega)$ is a generalised solution of

$$
\begin{aligned}
L v(x)+h(x, 0, \lambda) v(x) & =0 & & \text { in } \Omega, \\
v(x) & =0 & & \text { on } \partial \Omega
\end{aligned}
$$

Proof. For (b) $\Rightarrow$ (a) recall, by $[5$, Theorem 8.15$]$, that (b) implies $v \in L^{\infty}(\Omega)$. Hence 
$h(\cdot, 0, \lambda) v$ is essentially bounded on $\Omega$ and the continuity of $v$ on $\bar{\Omega}$ follows from Theorem 4.5 since $v=\operatorname{Th}(\cdot, 0, \lambda) v$.

We denote the differential operator in (5.2) by $L+A(\lambda)$, cf. (2.4). Since we are interested in positive solutions, we now give the following definition:

DefintTION 5.2. A number $\mu_{1}=\mu_{1}(\lambda)$ is called a principal eigenvalue for $L+A(\lambda)$ if there is a $v \in W_{0}^{1,2}(\Omega)$ such that

$$
\left.\begin{array}{rl}
v(x) & >0 \quad \text { for all } x \in \Omega \quad \text { and } \\
L v(x)+h(x, 0, \lambda) v(x) & =\mu_{1} v(x) \text { in } \Omega, \\
v(x) & =0 \text { on } \partial \Omega,
\end{array}\right\}
$$

in the generalised sense. (As shown in Lemma 5.1 such a weak eigenfunction is automatically in $C(\bar{\Omega})$.)

The existence of a principle eigenvalue for $L+A(\lambda)$ for all $\lambda \in \mathbb{R}$ can be established under either one of the following sets of conditions:

$$
\begin{aligned}
& L \text { is symmetric, i.e. } b^{i}=-c^{i} \text { for all } i=1, \ldots, n . \\
& a^{i j}, b^{i}, c^{i} \in C^{1}(\Omega) \text { and } D_{i} a^{i j}, D_{i} b^{i}, D_{i} c^{i} \in L^{\infty}(\Omega) \text { for } 1 \leqq i, j \leqq n .
\end{aligned}
$$

For proofs, we refer to [5, Chapter 8.12] in the case of (5.4), and to the recent paper [2] for the conditions (5.5).

REMARK 5.3. Using the $L^{p}$-theory, in particular [5, Theorem 9.30], conditions (5.5) on the coefficients of $L$ and (2.3) on $h$ imply that the positive eigenfunction $v$ associated with the principal eigenvalue $\mu_{1}$ has the regularity required in [2]: $v$ belongs to $W_{1 \text { loc }}^{2, p}(\Omega)$ for all finite $p>n$.

Additional properties of a principal eigenvalue $\mu_{1}$ are the following:

$\mu_{1}$ is algebraically simple; and

$\operatorname{Re} \mu \leqq \mu_{1}(\lambda)$ for any other eigenvalue $\mu$ of $L+A(\lambda)$.

Furthermore, if $\mu_{1}$ is a principal eigenvalue of $L+A(\lambda)$, then $\mu_{1}$ is also a principal eigenvalue of the adjoint operator $L^{*}+A(\lambda)$, where $L^{*}$ is obtained by replacing the coefficients $b^{i}$ in the definition of $L$ by $-c^{i}$ and vice versa. We shall denote by $\varphi_{\lambda}$ and $\psi_{\lambda}$ eigenfunctions of $L+A(\lambda)$ and of $L^{*}+A(\lambda)$ associated with the principal eigenvalue $\mu_{1}(\lambda)$.

REMARK 5.4. For regular problems (i.e. sufficiently smooth boundary $\partial \Omega$ and coefficients of $L$ ) the existence of a principal eigenvalue having the properties listed above is a classical result due to Courant and Hilbert in the symmetric case, and to Krein and Rutman in the non-symmetric case.

Henceforth we assume that either set of conditions (5.4) or (5.5) are fulfilled. Accordingly, a necessary condition for bifurcation of positive solutions is:

there is some $\lambda_{0} \in \mathbb{R}$ such that the principal eigenvalue of $L+A\left(\lambda_{0}\right)$ vanishes, i.e. $\mu_{1}\left(\lambda_{0}\right)=0$. 
By virtue of Lemma 5.1, condition (5.6) implies that $D_{u} G\left(\lambda_{0}, 0\right)$ has a one-dimensional kernel spanned by $\varphi_{\lambda_{0}}: N\left(D_{u} G\left(\lambda_{0}, 0\right)\right)=\operatorname{span}\left\{\varphi_{\lambda_{0}}\right\}$.

We now take up sufficient conditions for local bifurcation of nontrivial solutions of $(4.18)$ at $\left(\lambda_{0}, 0\right)$. We provide two alternative routes - the well-known transversality condition of Crandall and Rabinowitz [4], and the more general odd-crossing condition introduced in [7]. We begin with the former. In this case we require the additional differentiability assumption (2.5), which, together with Proposition 2.3, ensures that $D_{\lambda} D_{u} G(\lambda, 0) v$ exists and is given by $-T(\partial h / \partial \lambda(\cdot, 0, \lambda) v)$.

For convenience, we define the nontrivial solution set

$$
N S=\{(\lambda, u) \in \mathbb{R} \times C(\bar{\Omega}), G(\lambda, u)=0 \text { and } u \neq 0\} .
$$

Proposition 5.5. Assume (5.7) and that

$$
\frac{\partial h}{\partial \lambda}\left(x, 0, \lambda_{0}\right) \geqq 0 \text { and }>0 \text { in a subset of positive measure in } \Omega \text {. }
$$

Then there is an open neighbourhood $W$ of $\left(\lambda_{0}, 0\right)$ in $\mathbb{R} \times C(\bar{\Omega})$, and there exist continuous functions $\lambda: J \rightarrow \mathbb{R}$ and $z: J \rightarrow C(\bar{\Omega})$, where $J$ is an open interval in $\mathbb{R}$ containing 0 , such that $\lambda(0)=\lambda_{0}, z(0)=0$ and locally the unique nontrivial solution set is given by

$$
N S \cap W=\left\{\left(\lambda(s), s\left(\varphi_{\lambda_{0}}+z(s)\right)\right), s \in J \text { and } s \neq 0\right\} .
$$

Proof. Following the development in [4], it is enough to show that

$$
D_{\lambda} D_{u} G\left(\lambda_{0}, 0\right) \varphi_{\lambda_{0}} \notin R\left(D_{u} G\left(\lambda_{0}, 0\right)\right) \text {. }
$$

Now for $u \in C(\bar{\Omega})$,

$$
D_{\lambda} D_{u} G\left(\lambda_{0}, 0\right) u \in R\left(D_{u} G\left(\lambda_{0}, 0\right)\right)
$$

$\Leftrightarrow$ there is some $v \in C(\bar{\Omega})$ such that $D_{u} G\left(\lambda_{0}, 0\right) v=D_{\lambda} D_{u} G\left(\lambda_{0}, 0\right) u$

$\Leftrightarrow$ there is some $v \in C(\bar{\Omega})$ such that $v-T A\left(\lambda_{0}\right) v=-T\left(\partial h / \partial \lambda\left(\cdot, 0, \lambda_{0}\right) u\right)$

$\Leftrightarrow$ there is some $v \in W_{0}^{1,2}(\Omega)$ such that $v$ is a generalised solution of

$$
L v+A\left(\lambda_{0}\right) v=\partial h / \partial \lambda\left(\cdot, 0, \lambda_{0}\right) u
$$

$\Leftrightarrow \int_{\Omega} \partial h / \partial \lambda\left(x, 0, \lambda_{0}\right) u(x) \psi_{\lambda_{0}}(x) d x=0$,

by [5, Theorem 8.6], where $\psi_{\lambda_{0}}$ is a positive principal eigenfunction of $L^{*}+A\left(\lambda_{0}\right)$. However, by virtue of (5.9) and the positivity of $\varphi_{\lambda_{0}}$ and $\psi_{\lambda_{0}}$, we find that

$$
\int_{\Omega} \frac{\partial h}{\partial \lambda}\left(x, 0, \lambda_{0}\right) \varphi_{\lambda_{0}}(x) \psi_{\lambda_{0}}(x) d x>0
$$

and thus (5.11) follows.

Using the results of [7], we can eliminate the differentiability condition (2.5), and generalise condition (5.9) to a monotonicity property of $\lambda \mapsto h(x, 0, \lambda)$ (see (5.13) below). However, in this case we generally have nonuniqueness of local nontrivial solution branches.

To formulate a weaker version of $(5.10)$, let

$$
\begin{aligned}
& K_{\varepsilon}^{+}=\left\{s v, s>0 \text { and } v \in B_{\varepsilon}\left(\varphi_{\lambda_{0}}\right)\right\} \text { and } K_{\varepsilon}^{-}=-K_{\varepsilon}^{+}, \\
& \text {where } 0<\varepsilon<\left\|\varphi_{\lambda_{0}}\right\|_{\infty} \text { and } \\
& B_{\varepsilon}\left(\varphi_{\lambda_{0}}\right)=\left\{v \in C(\bar{\Omega}):\left\|v-\varphi_{\lambda_{0}}\right\|_{\infty}<\varepsilon\right\} .
\end{aligned}
$$


Proposition 5.6. Assume (5.7) and that

$$
\begin{aligned}
& h(x, 0, \cdot):\left(\lambda_{0}-\delta, \lambda_{0}+\delta\right) \rightarrow \mathbb{R} \text { is monotone for some } \delta>0 \\
& \text { and for all } x \in \Omega \text { and strictly monotone for all } x \text { in a subset of } \\
& \text { positive measure in } \Omega .
\end{aligned}
$$

Then there exist $\varepsilon \in\left(0, \frac{1}{2}\left\|\varphi_{\lambda_{0}}\right\|_{\infty}\right]$ and an open neighbourhood $W(\varepsilon)$ of $\left(\lambda_{0}, 0\right)$ in $\mathbb{R} \times C(\bar{\Omega})$ such that $\left|\lambda_{0}-\lambda\right|+\|u\|_{\infty}<\varepsilon$ for all $(\lambda, u) \in W(\varepsilon)$ and $\left(N S \cup\left\{\left(\lambda_{0}, 0\right)\right\}\right) \cap W(\varepsilon)$ is a connected subset of $N S \cup\left\{\left(\lambda_{0}, 0\right)\right\}$ for the $\mathbb{R} \times C(\overline{\mathbf{\Omega}})$ topology. Furthermore, setting $N S_{\varepsilon}^{ \pm}=(N S \cap W(\varepsilon)) \cap\left(\left(\lambda_{0}-\varepsilon, \lambda_{0}+\varepsilon\right) \times K_{\varepsilon}^{ \pm}\right)$we have $N S \cap W(\varepsilon)=N S_{\varepsilon}^{+} \cup N S_{\varepsilon}^{-}$and $N S_{\varepsilon}^{ \pm} \neq \varnothing$.

Proof. Assumption (5.13) implies that the function $\left(h\left(\cdot, 0, \lambda_{0}\right)-h(\cdot, 0, \lambda)\right) \varphi_{\lambda_{0}}$ is nonnegative (or nonpositive) and for $\lambda \in\left(\lambda_{0}-\delta, \lambda_{0}+\delta\right) \backslash\left\{\lambda_{0}\right\}$ it is positive (or negative) in a subset of $\Omega$ of positive measure. The same arguments as in the proof of Proposition 5.5 then yield that

$$
T\left(A\left(\lambda_{0}\right)-A(\lambda)\right) \varphi_{\lambda_{0}} \notin R\left(D_{u} G\left(\lambda_{0}, 0\right)\right) \quad \text { for } \lambda \in\left(\lambda_{0}-\delta, \lambda_{0}+\delta\right) \backslash\left\{\lambda_{0}\right\},
$$

where we used again Definition (5.2). In the sequel, we denote the function $T\left(A\left(\lambda_{0}\right)-A(\lambda)\right) \varphi_{\lambda_{0}}$ by $B(\lambda) \varphi_{0}$. By the Definition 4.4 of $T$ this function solves

$$
\begin{aligned}
L\left(B(\lambda) \varphi_{\lambda_{0}}\right)+\left(h\left(\cdot, 0, \lambda_{0}\right)-h(\cdot, 0, \lambda)\right) \varphi_{\lambda_{0}}=0 & \text { in } \Omega, \\
B(\lambda) \varphi_{\lambda_{0}}=0 & \text { on } \partial \Omega
\end{aligned}
$$

in the generalised sense. Recall, in particular, that by Theorem 4.5 the homogeneous Dirichlet boundary conditions are satisfied pointwise in the classical sense. By the strong maximum principle ([5, Theorem 8.19$])$, it then follows that

$$
\begin{aligned}
& B(\lambda) \varphi_{\lambda_{0}} \text { is non-zero in } \Omega \text { for } \lambda \in\left(\lambda_{0}-\delta, \lambda_{0}+\delta\right) \backslash\left\{\lambda_{0}\right\} \\
& \text { and changes sign when } \lambda \text { passes } \lambda_{0} .
\end{aligned}
$$

Local bifurcation of a continuum is established when we have shown that the family $D_{u} G(\lambda, 0)=I-T A(\lambda)$ has an odd crossing number at $\lambda=\lambda_{0}$, cf. [7]. This, in turn, is equivalent to a change of sign of the derivative, denoted $\Phi_{v}(\lambda, 0)$, of a scalar bifurcation function $\Phi(\lambda, v)$ with respect to the real variable $v$ that parametrises the kernel $N\left(D_{u} G\left(\lambda_{0}, 0\right)\right)=\operatorname{span}\left[\varphi_{\lambda_{0}}\right]$ (see [7, Theorem 3.1]). Furthermore, such a change of sign is independent of the choice of $\Phi$, i.e. independent of the choice of a complementary space for $R\left(D_{u} G\left(\lambda_{0}, 0\right)\right)$, provided that the orientation is preserved, cf. [7, Theorem 3.1]. For fixed $\lambda \in\left(\lambda_{0}-\delta, \lambda_{0}+\delta\right) \backslash\left\{\lambda_{0}\right\}$, we choose the complementing space $E=\operatorname{span}\left\{\left|B(\lambda) \varphi_{\lambda_{0}}\right|\right\}$ (see $\left.(5.15)\right)$. The projection of $C(\bar{\Omega})$ onto $E$ along $R\left(D_{u} G\left(\lambda_{0}, 0\right)\right)$ is then given by

$$
(I-Q) v=\left(\int_{\Omega}\left|B(\lambda) \varphi_{\lambda_{0}}\right| \psi_{\lambda_{0}} d x\right)^{-1}\left(\int_{\Omega} v \psi_{\lambda_{0}} d x\right)\left|B(\lambda) \varphi_{\lambda_{0}}\right|, v \in C(\bar{\Omega})
$$

where the left-hand side of the equation coincides with that of [7]. Formula (1.14) 
then shows that

$$
\operatorname{sign} \Phi_{v}(\lambda, 0)=\operatorname{sign} \int_{\Omega} B(\lambda) \varphi_{\lambda_{0}} \psi_{\lambda_{0}} d x
$$

which, in view of (5.17), yields a change of sign of $\Phi_{v}(\lambda, 0)$ at $\lambda=\lambda_{0}$.

All solutions of $G(\lambda, u)=0$ in $N S \cap W$ for some open neighbourhood $W$ of $\left(\lambda_{0}, 0\right)$ are obtained by the method of Lyapunov and Schmidt and they have the form

$$
\begin{aligned}
& \left(\lambda, s \varphi_{\lambda_{0}}+w(\lambda, s)\right) \text { where }(\lambda, s) \text { is in a } \\
& \text { neighbourhood } \tilde{W} \text { of }\left(\lambda_{0}, 0\right) \in \mathbb{R}^{2}, w: \tilde{W} \rightarrow C(\bar{\Omega}), \\
& \text { and } \Phi(\lambda, s)=0
\end{aligned}
$$

(cf. e.g. [7]). Since $\Phi_{s}(\lambda, 0)$ changes sign at $\lambda=\lambda_{0}$, the intermediate value theorem yields a continuum $\left(N S \cup\left\{\left(\lambda_{0}, 0\right)\right\}\right) \cap W$. Finally, since $w(\lambda, 0)=0$ and since the continuous partial derivative $w_{s}$ satisfies $w_{s}\left(\lambda_{0}, 0\right)=0$ (see [7, Chapter 1]), we get $(\lambda, u)=\left(\lambda, s \varphi_{\lambda_{0}}+w(\lambda, s)\right) \in\left(\lambda_{0}-\varepsilon, \lambda_{0}+\varepsilon\right) \times K_{\varepsilon}^{+}\left(K_{\varepsilon}^{-}\right)$provided $(\lambda, u)$ is in some suitable neighbourhood $W(\varepsilon)$ of $\left(\lambda_{0}, 0\right) \in \mathbb{R} \times C(\bar{\Omega})$ and $s>0(s<0)$.

REMARK 5.7. We emphasise that in spite of the algebraic simplicity of the principal eigenvalue $\mu_{1}=0$ of $L+A\left(\lambda_{0}\right)$ (see (5.6) and (5.7), the eigenvalue 0 of $D_{u} G\left(\lambda_{0}, 0\right)$ is not necessarily algebraically simple. Recall that the eigenvalue $\mu_{1}=0$ of $L+A\left(\lambda_{0}\right)$ is said to be algebraically simple if

$$
\begin{aligned}
L v+h\left(\cdot, 0, \lambda_{0}\right) v & =\varphi_{\lambda_{0}} & & \text { in } \Omega, \\
v & =0 & & \text { on } \partial \Omega,
\end{aligned}
$$

has no solution $v \in W_{0}^{1,2}(\Omega)$ in the generalised sense. On the other hand, by the definition of $G$, the eigenvalue 0 of $D_{u} G\left(\lambda_{0}, 0\right)$ is algebraically simple if

$$
\begin{aligned}
L v+h\left(\cdot, 0, \lambda_{0}\right) v & =-h\left(\cdot, 0, \lambda_{0}\right) \varphi_{\lambda_{0}} & & \text { in } \Omega, \\
v & =0 & & \text { on } \partial \Omega,
\end{aligned}
$$

has no solution $v \in W_{0}^{1,2}(\Omega)$ in the generalised sense (see also Lemma 5.1). Clearly the solvability of problem (5.22) is not generally equivalent to that of (5.21).

REMARK 5.8. By a famous result of Rabinowitz [9] and its generalisations [7], we obtain a global conclusion concerning bifurcation at $\lambda_{0}$ provided that the LeraySchauder degree of $u \mapsto G(\lambda, u)=u-K(\lambda, u)$ (where $K$ is compact) changes sign as $\lambda$ crosses $\lambda_{0}$ along the branch of trivial solutions, $\{(\lambda, 0)\}$. In Proposition 5.5 the hypotheses (5.7) and (5.9) imply that the transversality condition (5.11) is satisfied, and, as is well-known [7], this ensures that the degree changes sign as $\lambda$ crosses $\lambda_{0}$. Referring to [7], we see that this change of sign also occurs under the weaker assumptions of Proposition 5.6. We stress that the above conditions imply global bifurcation at $\lambda_{0}$ without invoking directly the algebraic multiplicity of the zero eigenvalue of $D_{u} G\left(\lambda_{0}, 0\right)$.

Considering $N S \cup\left\{\left(\lambda_{0}, 0\right)\right\}$ as a subspace of the metric space $\mathbb{R} \times C(\bar{\Omega})$, let $C$ denote the component of $N S \cup\left\{\left(\lambda_{0}, 0\right)\right\}$ containing $\left(\lambda_{0}, 0\right)$.

Proposition 5.9. Under the hypotheses of either Proposition 5.5 or Proposition 5.6, $C$ has at least one of the following properties: 
(i) $C$ is unbounded with respect to the $\mathbb{R} \times C(\bar{\Omega})$ topology.

(ii) $\bar{C}$ (the closure of $C$ in $\mathbb{R} \times C(\bar{\Omega})$ ) contains an element $(\lambda, 0)$ with $\lambda \neq \lambda_{0}$.

We now come to our main result in this section. Let $C^{ \pm}$denote the union of all components of $C \backslash\left\{\left(\lambda_{0}, 0\right)\right\}$ that contain elements of $N S_{\varepsilon}^{ \pm}$where $\varepsilon$ is sufficiently small so that Proposition 5.6 holds. Under the hypotheses of Proposition 5.5, $\mathrm{C}^{+}\left(\mathrm{C}^{-}\right)$ contains all local solutions of the form $\left\{\left(\lambda(s), s\left(\varphi_{\lambda_{0}}+z(s)\right), s \in J\right.\right.$ and $\left.s>0(s<0)\right\}$. In general, $C^{ \pm}$are independent of the choice of $\varepsilon>0$.

THEOREM 5.10. Under the hypotheses of this section:

(i) $C^{+} \subset P^{+} \equiv\{(\lambda, u) \in \mathbb{R} \times C(\bar{\Omega}), u(x)>0$ in $\Omega\}$;

(ii) $C^{-} \subset P^{-} \equiv\{(\lambda, u) \in \mathbb{R} \times C(\bar{\Omega}), u(x)<0$ in $\Omega\}$;

(iii) $C=C^{+} \cup C^{-} \cup\left\{\left(\lambda_{0}, 0\right)\right\}$.

Moreover, if we assume that

the principal eigenvalue $\mu_{1}(\lambda)$ of $L+A(\lambda)$ vanishes if and only if $\lambda=\lambda_{0}$, then

(iv) $\bar{C} \cap(\mathbb{R} \times\{0\})=\left\{\left(\lambda_{0}, 0\right)\right\}$ and $C^{+}$and $C^{-}$are each unbounded subsets of $\mathbb{R} \times C(\bar{\Omega})$.

Proof. If we adopt the hypotheses of Proposition 5.5, then $\mathrm{C}^{+}$is connected, and the proof of (i) amounts to demonstrating that

(a) $C^{+} \cap P^{+}=\varnothing$,

(b) $C^{+} \cap P^{+}$is closed relative to $C^{+}$,

and

(c) $C^{+} \cap P^{+}$is open relative to $C^{+}$.

On the other hand, Proposition 5.6 does not necessarily imply that $C^{+}$is connected, in which case (by an abuse of notation) $C^{+}$in (5.24) may actually represent a component of $C^{+}$(a maximal connected subset of $C^{+}$).

Obviously once (5.24) is established, (i) then follows by taking the union of all components of $C^{+}$.

(a) Using our earlier notation, let $(\lambda, u) \in N S_{\varepsilon}^{+}$. Then there is some $s(u)>0$ and $z(u) \in B_{\varepsilon}(0)$ such that $u=s(u)\left(\varphi_{\lambda_{0}}+z(u)\right)$. Furthermore $\varepsilon>\|u\|_{\infty} \geqq s(u)\left(\left\|\varphi_{\lambda_{0}}\right\|_{\infty}-\right.$ $\left.\|\mathrm{z}(\mathrm{u})\|_{\infty}\right) \geqq \mathrm{s}(\mathrm{u})\left(\left\|\varphi_{\lambda_{0}}\right\|_{\infty}-\varepsilon\right) \geqq \frac{1}{2} \mathrm{~s}(\mathrm{u})\left\|\varphi_{\lambda_{0}}\right\|_{\infty}$ and so $\mathrm{s}(u) \rightarrow 0$ and $\|z(u)\|_{\infty} \rightarrow 0$ as $\varepsilon \rightarrow 0$. Also $\varphi_{\lambda_{0}}+z(u) \in W_{0}^{1,2}(\Omega) \cap C(\bar{\Omega})$ is a generalised solution of

$$
L\left(\varphi_{\lambda_{0}}+z(u)\right)+h(\cdot, u, \lambda)\left(\varphi_{\lambda_{0}}+z(u)\right)=0 \text { in } \Omega,
$$

and there is an $M>0$ such that $\|h(\cdot, u, \lambda)\|_{\infty} \leqq M$ for all $(\lambda, u) \in N S_{\varepsilon}^{+}$. Hence, by Theorem 3.2 there is some constant $\gamma>0$ such that for any $(\lambda, u) \in N S_{\varepsilon}^{+}$, either $\varphi_{\lambda_{0}}+z(u)>0$ in $\Omega$ or meas $\mathscr{D}(u) \geqq \gamma$ where $\mathscr{D}(u)=\left\{x \in \Omega:\left(\varphi_{\lambda_{0}}+z(u)\right)(x)<0\right\}$. For $\delta>0$, set $M(\delta)=\{x \in \Omega, d(x, \partial \Omega)<\delta\}$. Since $M(\delta)$ is an open set, its characteristic function $\chi_{\delta}$ is measurable and $0 \leqq \chi_{\delta} \leqq 1$ on $\Omega$. Furthermore, for all $x \in \Omega$, $\lim _{\delta \rightarrow 0} \chi_{\delta}(x)=0$ and so by dominated convergence $\lim _{\delta \rightarrow 0}$ meas $M(\delta)=0$. But for each $\delta>0, \Omega \backslash M(\delta)$ is compact and there is some $\mu(\delta)>0$ such that $\varphi_{\lambda_{0}}(x) \geqq \mu(\delta)$ for all $x \in \Omega \backslash M(\delta)$. Now for $x \in \mathscr{D}(u), \quad \varphi_{\lambda_{0}}(x)=\varphi_{\lambda_{0}}(x)+z(u)(x)-z(u)(x)<$ $-z(u)(x) \leqq\|z(u)\|_{\infty}<\varepsilon$. Hence for all $\delta>0, \mathscr{D}(u) \subset M(\delta)$ if $0<\varepsilon<\mu(\delta)$. Choosing $\delta$ so small that meas $M(\delta)<\gamma$, we conclude that $\varphi_{\lambda_{0}}+z(u)>0$ in $\Omega$ whenever 
$(\lambda, u) \in N S_{\varepsilon}^{+}$and $0<\varepsilon<\mu(\delta)$. But $N S_{\varepsilon}^{+} \neq \varnothing$ by Propositions 5.5 and 5.6 and we have just shown that $N S_{\varepsilon}^{+} \subset C^{+} \cap P^{+}$for $0<\varepsilon<\mu(\delta)$.

(b) Suppose there is some $(\lambda, u) \in C^{+}$and some sequence $\left(\left(\lambda_{n}, u_{n}\right)\right)$ in $C^{+} \cap P^{+}$such that $\lambda_{n} \rightarrow \lambda$ and $\left\|u_{n}-u\right\|_{\infty} \rightarrow 0$. Then $u \geqq 0$ in $\Omega$. Since

$$
L u+h(\cdot, u, \lambda)^{-} u=-h(\cdot, u, \lambda)^{+} u \leqq 0 \quad \text { in } \Omega
$$

in the generalised sense, it follows from [5, Theorem 8.19] that either $u>0$ in $\Omega$ or $u \equiv 0$ in $\Omega$. However, by definition, $C^{+}$does not contain a trivial solution, and thus $(\lambda, u) \in C^{+} \cap P^{+}$.

(c) If $C^{+} \cap P^{+}$is not open relative to $C^{+}$, then there is some point $(\lambda, u) \in C^{+} \cap P^{+}$ and a sequence $\left(\left(\lambda_{n}, u_{n}\right)\right)$ in $C^{+} \backslash P^{+}$such that $\lambda_{n} \rightarrow \lambda$ and $\left\|u_{n}-u\right\|_{\infty} \rightarrow 0$. The functions $u_{n} \in W_{0}^{1,2}(\Omega) \cap C(\bar{\Omega})$ are generalised solutions of

$$
L u_{n}+h\left(\cdot, u_{n}, \lambda_{n}\right)=0 \text { in } \Omega
$$

and by the convergence of $\left(\lambda_{n}, u_{n}\right)$ in $\mathbb{R} \times C(\bar{\Omega})$ there is some constant $M$, such that $\left\|h\left(\cdot, u_{n}, \lambda_{n}\right)\right\|_{\infty} \leqq M$ for all $n \in \mathbb{N}$. Then by Theorem 3.2, there is some constant $\gamma>0$ such that for any $n \in \mathbb{N}$, either $u_{n}>0$ in $\Omega$ or meas $\left(\mathscr{D}_{n}\right) \geqq \gamma$ where $\mathscr{D}_{n}=$ $\left\{x \in \Omega, u_{n}(x)<0\right\}$. Since $u>0$ in $\Omega$ and $\left\|u_{n}-u\right\|_{\infty} \rightarrow 0$, the arguments given in the proof of (a), however, yield meas $\mathscr{D}_{n} \rightarrow 0$ as $n \rightarrow \infty$. Thus, there is some $n_{0} \in \mathbb{N}$ such that $u_{n}>0$ in $\Omega$, i.e. $\left(\lambda_{n}, u_{n}\right) \in C^{+} \cap P^{+}$for all $n \geqq n_{0}$, contradicting the assumption on the sequence $\left(\left(\lambda_{n}, u_{n}\right)\right)$.

Obviously, the proof of (ii) is nearly identical to that of (i), and (iii) is a trivial consequence of the definition: $C \backslash\left\{\left(\lambda_{0}, 0\right)\right\}=C^{+} \cup C^{-}$. Finally, to establish (iv), let $\left\{\left(\lambda_{n}, u_{n}\right)\right\}$ be a sequence contained in $C \backslash\left\{\left(\lambda_{0}, 0\right)\right\}$ such that $\lambda_{n} \rightarrow \lambda$ and $\left\|u_{n}\right\|_{\infty} \rightarrow 0$. By (iii) we may assume that $\left(\lambda_{n}, u_{n}\right) \in P^{+}$for all $\tilde{n}$. Setting $v_{n}=u_{n} /\left\|u_{n}\right\|_{\infty}$, and employing an argument identical to that in [10, Lemma 2.7], we find that $v_{n} \rightarrow v$ in $C(\bar{\Omega})$, $\|v\|_{\infty}=1$, and

$$
v=T h(\cdot, 0, \lambda) v=T A(\lambda) v .
$$

Since $v_{n}>0$ in $\Omega$, it then follows from Lemma 5.1 that $v \geqq 0$ in $\Omega$ is a generalised solution of (5.2). But by the same observation made in (3.9), we conclude that $v>0$ in $\Omega\left(\|v\|_{\infty}=1\right)$. Thus, $L+A(\lambda)$ has principal eigenvalue 0 , and the first part of (iv) follows from (5.23). The unboundedness of $C^{+}\left(C^{-}\right)$is a consequence of [9, Theorem 1.27] and $C^{+} \subset P^{+}\left(C^{-} \subset P^{-}\right)$.

\section{Continuation}

In this section we consider problem (4.18) assuming that

$$
f(x, 0,0)=g(x, 0)=0 \text { for almost all } x \in \Omega,
$$

and also under the hypotheses of Sections 2 and 4 (except (2.5)). Thus, $G(0,0)=0$, i.e. in contrast to Section 5, we have the a priori existence of merely one solution point $(0,0) \in \mathbb{R} \times C(\bar{\Omega})$. Now if

$$
D_{u} G(0,0)=I-T A(0): C(\bar{\Omega}) \rightarrow C(\bar{\Omega}) \text { is invertible, }
$$

then there is a local, unique curve of solutions of $(4.18)$ containing $(0,0)$, by virtue of the implicit function theorem. From the Riesz-Schauder theory and Lemma 5.1, 
it follows that condition (6.2) is equivalent to:

$$
\begin{aligned}
& v=0 \text { is the only solution of } \\
& L v+h(\cdot, 0,0) v=0 \text { in } \Omega, \\
& v=0 \text { on } \partial \Omega,
\end{aligned}
$$

in the generalised sense. A sufficient condition for this is the following lemma:

LEMMA 6.1. If

$$
h(x, 0,0) \leqq 0 \quad \text { for almost all } x \in \Omega,
$$

then (6.3) and hence, (6.2) hold.

Proof. The operator $L+h(\cdot, 0,0)$ satisfies all three conditions in (2.2). Hence, (6.3) follows from a routine application of the maximum (and minimum) principle (cf. [5, Corollary 8.2]).

By virtue of the implicit function theorem, we now have

Proposition 6.2. If (6.4) holds, then there is a unique local branch of solution of (4.18) of the form

$$
L S=\{(\lambda, \tilde{u}(\lambda)),|\lambda|<\varepsilon\}
$$

where $\tilde{u}:(-\varepsilon, \varepsilon) \rightarrow C(\bar{\Omega})$ is continuous, $\tilde{u}(0)=0$, and $G(\lambda, \tilde{u}(\lambda))=0$ for all $|\lambda|<\varepsilon$.

In order to establish the main results of this section, we require the following sign conditions upon the functions $g$ and $h$ (the latter of which implies (6.4)):

$$
\begin{aligned}
& \text { (i) for each } \lambda \geqq 0(\leqq 0), g(x, \lambda) \geqq 0(\leqq 0) \text {, } \\
& \text { for almost all } x \in \Omega \text {, and } g(x, \lambda) \neq 0 \text { in a } \\
& \text { subset of positive measure in } \Omega \text { if } \lambda \neq 0 \text {; } \\
& \text { (ii) } h(x, s, 0) \leqq 0 \\
& \text { for all } s \in \mathbb{R} \text { and almost all } x \in \Omega \text {. }
\end{aligned}
$$

Apart from these conditions on $f(x, s, \lambda)=g(x, \lambda)+h(x, s, \lambda) s$ along the axes of the $(s, \lambda)$-plane, we also need one of the following conditions in a neighbourhood of $(0,0)$ : There exists some $\delta>0$ such that

$$
\left.\begin{array}{l}
\text { either } \\
\text { (i) } h(x, s, \lambda) \leqq 0 \\
\text { or } \\
\text { (ii) } h(x, s, \lambda) s \geqq 0, \\
\text { for }|s|<\delta,|\lambda|<\delta \text { and for almost all } x \in \Omega \text {. }
\end{array}\right\}
$$

By a now-standard application of the Leray-Schauder degree, the local solution curve $L S$ is part of a global branch of solutions of (4.18), denoted by $C$, subject to an alternative described as follows (cf. $[1,9]$ ): Let

$$
S=\{(\lambda, u) \in \mathbb{R} \times C(\bar{\Omega}): G(\lambda, u)=0\}
$$


be the set of all solutions of (4.18). Define $C^{+}\left(C^{-}\right)$as the component of $S \backslash\{(0,0)\}$ that contains $L S^{+}\left(L S^{-}\right)=\{(\lambda, \tilde{u}(\lambda)), 0<\lambda<\varepsilon(-\varepsilon<\lambda<0)\}$. Then either

$$
\begin{aligned}
& \text { (i) } C^{+} \text {and } C^{-} \text {are unbounded in } \mathbb{R} \times C(\bar{\Omega}) \\
& \text { or } \\
& \text { (ii) } C^{+} \cap C^{-} \neq \varnothing \text {. }
\end{aligned}
$$

However, our assumptions (6.1) and (6.6) rule out (6.9(ii)). Indeed, $(0, u) \in S$ is equivalent to the statement that $u \in W_{0}^{1,2}(\Omega)$ is a generalised solution of

$$
L u(x)+f(x, u(x), 0)=0 \quad \text { in } \Omega .
$$

But by (6.1) and (6.6), $f(x, u(x), 0)=h(x, u(x), 0) u(x)$ and $h(x, u(x), 0) \leqq 0$ almost everywhere on $\Omega$. It follows that the operator $L+h(\cdot, u, 0)$ satisfies the conditions (2.2) and consequently that $S \cap(\{0\} \times C(\bar{\Omega}))=\{(0,0)\}$. Therefore the components $\mathrm{C}^{+}$and $C^{-}$are separated by the hyperplane $\{0\} \times C(\bar{\Omega})$ in $\mathbb{R} \times C(\bar{\Omega})$ and by (6.9) the sets $C^{+}$and $C^{-}$are both unbounded connected subsets of $S$. We now give our main result of this section.

THEOREM 6.3. Under the hypotheses of this section,

(i) $C^{+} \subset P^{+}$, (ii) $C^{-} \subset P^{-}$, (iii) $C=C^{+} \cup C^{-} \cup\{(0,0)\}$, where $P^{ \pm}$are defined as in Theorem 5.10.

Proof. We prove only part (i) by establishing properties (5.24(a)-(c)) as in the proof of Theorem 5.10. The arguments are obviously similar, and we give only the new aspects.

(a) A solution $(\lambda, \tilde{u}(\lambda)) \in L S^{+}$fulfils

$$
\begin{aligned}
L \tilde{u}(\lambda)+h(\cdot, \tilde{u}(\lambda), \lambda) \tilde{u}(\lambda)=-g(\cdot, \lambda) \leqq 0 & & \text { in } \Omega, \\
\tilde{u}(\lambda)=0 & & \text { on } \partial \Omega,
\end{aligned}
$$

in the generalised sense. If $\|\tilde{u}(\lambda)\|_{\infty}<\delta$ and $0<\lambda<\delta$, then assumption (6.7(i)) implies that $h(\cdot, \tilde{u}(\lambda), \lambda) \leqq 0$ in $\Omega$. Since the linear operator $\hat{L}=L+h(\cdot, \tilde{u}(\lambda), \lambda)$ satisfies all three conditions in (2.2), it follows from the strong maximum principle that either $\tilde{u}(\lambda)>0$ or $\tilde{u}(\lambda) \equiv 0$ in $\Omega$. However, this second alternative is ruled out by virtue of (6.6(i)) and (6.11). Therefore $(\lambda, \tilde{u}(\lambda)) \in P^{+}$and $C^{+} \cap P^{+} \neq \varnothing$. For the case (6.7(ii)), we have $L \tilde{u}(\lambda) \leqq 0$ in $\Omega$, and we draw the same conclusion.

(b) Assume $(\lambda, u) \in C^{+}$and a sequence $\left(\left(\lambda_{u}, u_{n}\right)\right) \subset C^{+} \cap P^{+}$such that $\lambda_{n} \rightarrow \lambda$ and $\left\|u_{n}-u\right\|_{\infty} \rightarrow 0$. Then $\lambda>0$ and $u(x) \geqq 0$ on $\Omega$. Since $u \in W_{0}^{1,2}(\Omega)$ solves

$$
L u+h(\cdot, u, \lambda)^{-} u=-h(\cdot, u, \lambda)^{+} u-g(\cdot, \lambda) \leqq 0 \quad \text { in } \Omega
$$

in the generalised sense, the rest of the argument identical to the one for (b) given in the proof of Theorem 5.10.

(c) Here the argument is again similar to that given in the proof of Theorem 5.10. Observe that, instead of (5.27), we now have

$$
L u_{n}+h\left(\cdot, u_{n}, \lambda_{n}\right) u_{n}=-g\left(\cdot, \lambda_{n}\right) \leqq 0 \quad \text { in } \Omega,
$$

in the generalised sense, and Theorem 3.2 can again be applied. 


\section{Concluding remarks}

Before ending this work, we point out the direct applicability of the results of Section 6 to the anti-plane-shear problem of nonlinear elasticity (e.g. $[8,11]$ ). Let $\Omega \subset \mathbb{R}^{2}(n=2)$, and suppose that $\mathscr{B} \equiv \Omega \times[0, L] \subset \mathbb{R}^{3}$ is a stressfree configuration of an elastic body. Let $\{\boldsymbol{i}, \boldsymbol{j}, \boldsymbol{k}\}$ denote the standard orthonormal basis for $\mathbb{R}^{3}$. Referring to (1.1), (2.1-3), we have that $u\left(x_{1}, x_{2}\right) \boldsymbol{k}$ is the (anti-plane-shear) displacement of the body, and $f\left(x_{1}, x_{2}, u, \lambda\right)=g\left(x_{1}, x_{2}, \lambda\right)+h\left(x_{1}, x_{2}, u, \lambda\right) u$ is the component of the body force along $\boldsymbol{k}$, for all $\left(x_{1}, x_{2}, x_{3}\right) \in \mathscr{B}$. Since $g$ is independent of the displacement $u$, it is called a "dead" load, while the configuration-dependent loading $h \cdot u$ is said to be "live". Let $\boldsymbol{S}(\boldsymbol{x}) \in \mathbb{R}^{3 \times 3}$ denote the first Piola-Kirchhoff stress tensor at $\boldsymbol{x} \in \mathscr{B}$. We require part of the shear response to be linear elastic as follows:

$$
S_{3 i}=a^{i j} D_{j} u, i=1,2,(j=1,2) .
$$

Finally, we choose $b^{i}=c^{i}=d \equiv 0$. Then (1.1) expresses the component of (local) linear momentum balance along $\boldsymbol{k}$, and also specifies that the portion of the boundary, $\partial \Omega \times[0, L] \subset \partial \mathscr{B}$, is immobile. Of course there are two other linear-momentumbalance equations, but they are decoupled from (1.1). (Generally, other body forces along $i$ and $j$ are required to maintain the anti-plane-shear displacement, although cf. [8] for special cases in which they are not.)

We now discuss the basic hypotheses of Section 6. Assumption (6.1) says that no body forces are applied when the control parameter is zero. Conditions (6.4), (6.6(ii)) and either of (6.7) place physically reasonable restrictions upon the local sign of the live load, while (6.6(i)) is a natural restriction to place upon the dead load. For example, gravity loading fulfils condition (6.6(i)).

REMARK 7.1. At first glance it appears that the results of Section 5 on bifurcation should also be applicable to the anti-phase-shear problem. However, conditions (5.1) seem artificial and are hard to motivate with concrete examples.

REMARK 7.2. For technical reasons, we must restrict ourselves to linear elasticity (7.1), and more generally to semi-linear equations (1.1). Nonetheless, we are able to handle "bad" coefficients and inhomogeneities and rough boundaries. Although there is nothing wrong with (7.1) (the same is not true for general deformations of threedimensional elastic bodies), it is, more generally, nonlinear in $D u$, in which case (1.1) becomes quasilinear. It is interesting to note that Rabinowitz's original results [9] hold for quasilinear equations-but at the price of smooth constitutive laws and smooth boundaries.

\section{Acknowledgments}

We thank L. E. Fraenkel and P. Rosakis for stimulating discussions during various stages of this work.

The work of T. J. H. was supported in part by NSF Grant No. 9103254. The work of T. J. H. and H. K. was supported in part by NATO Grant No. CRG 920809. 


\section{References}

1 J. C. Alexander and J. A. Yorke. The implicit function theorem and global methods of cohomology. J. Funct. Anal. 21 (1976), 330-339.

2 H. Berestycki, L. Nirenberg and S. R. S. Varadhan. The principal eigenvalue and maximum principle for second order elliptic operators in general domains. Comm. Pure Appl. Math. (to appear).

3 H. Brezis. Analyse fonctionelle (Paris: Masson, 1983).

4 M. G. Crandall and P. H. Rabinowitz. Bifurcation from simple eigenvalues. J. Funct. Anal. 8 (1971), 321-340.

5 D. Gilbarg and N. S. Trudinger. Elliptic Partial Differential Equations of Second Order, 2nd edn (Berlin: Springer, 1983).

6 T. J. Healey and H. Kielhöfer. Preservation of nodal structure on global bifurcating solution branches of elliptic equations with symmetry. J. Differential Equations (to appear).

7 H. Kielhöfer. Multiple eigenvalue bifurcation for Fredholm operators. J. Reine Angew. Math. 358 (1985), 104-124.

8 J. K. Knowles. On finite anti-plane-shear for incompressible elastic materials. J. Austral. Math. Soc. Ser. $B 19(1976), 400-415$.

9 P. H. Rabinowitz. Some global results for nonlinear eigenvalue problems. J. Funct. Anal. 7 (1971), 487-513.

10 P. H. Rabinowitz. Some aspects of nonlinear eigenvalue problems. Rocky Mountain J. Math. 3 (1973), 161-202.

11 P. Rosakis. Compact zones of shear transformation in an anisotropic solid. J. Mech. Phys. Solids $\mathbf{4 0}$ (1992), 1163-1195.

12 J. Serrin. A symmetry problem in potential theory. Arch. Rational Mech. Anal. 43 (1971), 304-318.

13 G. Stampacchia. Le problème de Dirichlet pour les equations elliptiques du second ordre à coefficients discontinus. Ann. Inst. Fourier (Grenoble) 15 (1965), 189-258.

(Issued 29 April 1994) 